\title{
Is provisional stenting the effective option? The WIDEST study (Wiktor stent in de novo stenosis)
}

\author{
D S Fluck, P Chenu, P Mills, A Davies, J Street, E Paul, R Balcon, C A Layton, for the \\ Widest Trial Investigators' Group
}

\begin{abstract}
Aim-To compare the immediate and late outcomes of patients treated by a policy of routine stent implantation with routine balloon angioplasty and the use of stents only when an ideal result has not been obtained.

Methods-A nine centre, multinational, randomised study of 300 patients with coronary artery disease thought suitable for treatment of a single lesion by balloon angioplasty or stent implantation. Only new lesions in patients who had not undergone previous bypass surgery were included, and totally occluded vessels were excluded.

Results-The initial procedure was considered successful in $96 \%$ of patients. There was more complete angiographic restoration of luminal diameter in patients treated by elective stent (minimum lumen diameter (MLD) $2.68 \mathrm{~mm}$ for stent $v 2.27 \mathrm{~mm}$ for balloon; $\mathrm{p} \mathrm{<} \mathrm{0.007),} \mathrm{but} \mathrm{analy-}$ sis of the subgroup of balloon angioplasty patients who crossed over to stenting showed that they achieved similar results to the elective stent group. Late luminal loss was greater in stented patients than in those undergoing balloon angioplasty only, and by six months the angiographic benefit of stenting had disappeared (MLD $1.90 \mathrm{~mm}$ for stent group $v 2.00 \mathrm{~mm}$ for balloon angioplasty). Angiographic and clinical results in the balloon angioplasty group were assisted by the high crossover rate $(30.1 \%)$. Both groups had similar symptom relief, with $58.9 \%$ of patients improving by two or more angina grades. The need for further revascularisation was also similar in the two groups at one year $(18.2 \%$ in the stented group $v 17.1 \%$ in the balloon angioplasty group). Haemorrhagic complications at the local arterial entry site were more common than expected and were distributed equally between the patients receiving full anticoagulation and those receiving antiplatelet treatment only. The results of both Wiktor stent placement and balloon angioplasty were similar to the findings in the stent group in previous randomised studies (Benestent II, STRESS).

Conclusions-Provisional stenting appears to offer the same longer term outcome as elective stenting in this selected group of patients. Improvement in the results of conventional balloon angioplasty in the past 10 years means that a policy of obtaining an ideal result without the use of stents appears to be practicable in many of these patients, with consequent cost savings.

(Heart 2000;84:522-528)
\end{abstract}

Keywords: stent; balloon angioplasty; coronary angioplasty

Since the introduction of percutaneous coronary angioplasty in 1977 by Gruentzig et al, ${ }^{1}$ the high rate of restenosis has been a continuing problem which has not been solved despite trials of a multitude of pharmacological and physical methods. In the period between 1985 and 1993, restenosis rates for angioplasty ranged from $19 \%$ to $63 \% .^{2}$ The introduction of coronary stenting in 1986 raised the hope that a technique had finally been developed that would overcome the high rate of restenosis, but this was unfortunately premature. ${ }^{3}$ In 1994, two landmark studies showed a small but significant reduction in the angiographic restenosis rate with the use of elective coronary stenting compared with conventional balloon angioplasty. ${ }^{45}$ These beneficial results, together with numerous anecdotal and registry reports, have fuelled the exponential rise in elective coronary stent placement, with typical stenting rates in the United Kingdom of up to $85 \%$ (London Chest Hospital, unpublished data). However, the incidence of ischaemic events and clinical restenosis remains at an unwelcome level in both of the randomised studies.

Since these trials were reported, there have been further developments in stent design and also in the techniques and technology of balloon angioplasty. This has been coupled with greater insights into the mechanisms of restenosis, the significance of changes in minimum lumen diameter, both after dilatation or stenting and at follow up, and advances in the pharmacological management of platelet activity after angioplasty and stenting. These developments have cast doubt on the advisability of the high rates of stent implantation now being employed in many centres, ${ }^{6}$ with the suggestion that a policy of optimal angioplasty and provisional stenting (when the outcome of angioplasty is suboptimal or a complication occurs) may be preferable.

The two key randomised trials both used the Palmaz-Schatz stent, a stainless steel slotted tube design with a central hinge. Our purpose in this study was to compare elective coronary stent placement, using a tantalum wire stent (Wiktor, Medtronic Inc, Minneapolis, Minnesota, USA) with optimal balloon angioplasty and selective stenting, in patients undergoing single vessel angioplasty. The objective was to determine whether optimal angioplasty with stent placement for less than ideal results was as effective as elective stent placement when 
best current practice was employed in the performance of the angioplasty and in subsequent management.

\section{Methods}

PATIENT SELECTION

Patients were eligible for the study if they had symptomatic coronary artery disease suitable for balloon angioplasty and implantation of a 3.0 to $4.5 \mathrm{~mm}$ diameter tantalum wire stent (Wiktor GX) in a single lesion. Patients were excluded if they were in the acute phase of a myocardial infarct (within the last seven days), had undergone bypass surgery or angioplasty to the target vessel or lesion, or required multivessel angioplasty. Patients were also excluded if the vessel was occluded (thrombolysis in myocardial infarction (TIMI) grade 0 ), if thrombus was present, if the lesion required more than one stent, if the lesion was ostial, or if there was significant left main stem coronary disease. Patients were also excluded if they had contraindications to anticoagulation or aspirin treatment, if they were pregnant, if they were suffering from uncontrolled hypertension (systolic blood pressure $>159 \mathrm{~mm} \mathrm{Hg}$, diastolic blood pressure $>109 \mathrm{~mm} \mathrm{Hg}$ ), or if they were enrolled in another trial.

\section{RANDOMISATION}

Patients were assessed for eligibility, and informed consent was obtained. They were then randomly assigned by telephone using a central randomisation centre. Patients from each centre were randomised in a block design to ensure that similar numbers were assigned to each treatment at each centre. The appropriate intervention had to be performed within seven days of randomisation. A register was kept at each centre of all patients who conformed to the trial inclusion criteria and who were not randomised, together with the reason for their non-inclusion in the study.

\section{BALLOON ANGIOPLASTY AND STENT}

IMPLANTATION

Angioplasty was performed with conventional techniques. Aspirin treatment was mandatory and initially an accelerated warfarin regimen was recommended when elective stenting was planned. However, with the increasing use of alternative antiplatelet and antithrombotic interventions that became common practice as the trial progressed, centres were allowed to use their institutions' usual preoperative and postoperative regimens. This meant that the use of ticlopidine in preference to full anticoagulation increased throughout the first year of the study and virtually replaced anticoagulation; abciximab was used in one patient only. Operators were encouraged to aim for the best possible angioplasty result in those patients randomised to balloon dilatation alone. Crossover to stenting was permitted at the discretion of the operator if a complication such as significant vessel wall dissection or acute vessel occlusion occurred, or if the operator was dissatisfied with the final result of dilatation alone. In the latter case the operator was advised to attempt to improve the outcome without use of a stent, but had the free option to implant a stent if it was considered clinically desirable. The Wiktor GX stent was the chosen stent for those patients randomised to elective stenting. If there was a need for a further stent to be deployed following implantation of the initial stent, or if the patient crossed over from the dilatation only arm, then alternative stents could be used at the discretion of the operator.

CLINICAL AND ANGIOGRAPHIC FOLLOW UP

The patients were routinely reviewed at three, six, and 12 months to assess angina status and current drug treatment. Cardiac and clinical end points were recorded, and the patients underwent an exercise test using the standard Bruce protocol. At the six month follow up, all patients were scheduled for a further angiogram unless a repeat angiogram had been performed within the previous three months and was available for analysis, or where a second intervention had been performed. Therefore, three angiograms were obtained for each patient-one immediately before the intervention, one after the intervention, and one at six months after the procedure.

\section{QUANTITATIVE CORONARY ANGIOGRAPHIC}

\section{ANALYSIS}

Patients were examined angiographically before and after intervention and at the six months follow up. Quantitative analysis of the acquired angiograms (quantitative coronary angiography, QCA) was performed independently at a core laboratory (Heart Core BV Leiden, Netherlands) using the cardiovascular measurement system (QCA-CMS, MEDIS Medical Imaging Systems, Leiden, Netherlands), version 3.32. The basic algorithms have been described elsewhere. ${ }^{78}$ For $35 \mathrm{~mm}$ cine film applications, the lesions of interest in a selected frame were magnified optically on a high quality cine projector, converted into video format, and digitised by a frame grabber at a resolution of $512 \times 768 \times 8$ bits, and stored in memory of the QCA workstation. For a digital application with the images stored in DICOM format on a CD-R (compact disc recordable), the lesions of interest were extracted from the digital matrix data $(512 \times 512 \times 8$ bits $)$ and digitally zoomed to the correct matrix size using an interpolation approach.

The quantitative analysis done with the QCA-CMS analytical software is as follows. To select the coronary segment to be analysed, the user only needs to define the start and end point of that segment. In the next step, an arterial pathline through the segment of interest is computed automatically. ${ }^{8}$ The standard contour detection procedure is carried out in two iterations relative to a model. In the first iteration the detected pathline is the model. The individual left and right vessel contours detected in this first iteration function as models in the second iteration. To detect the contours, scanlines are defined perpendicular to the model. For each point or pixel along such scanlines the corresponding edge strength value (local change in brightness level) is computed as the weighted sum of the correspond- 
ing values of first and second derivative functions applied to the brightness values along these scanlines. The edge strength values are input to the minimum cost algorithm (MCA) contour detection algorithm, which searches for an optimal contour path along the entire segment. To correct for the limited resolution of the entire $x$ ray system, the MCA is modified in the second iteration based on an analysis of the point spread function of the imaging chain, which is of particular importance for the accurate measurement of small diameters, as in coronary obstructions.

As the Wiktor GX stent is radio-opaque, the standard MCA will often not be able to follow the luminal contrast boundaries in between the stent struts. For that reason the MCA procedure is followed by the application of the gradient field transform (GFT) edge detection algorithm, which has been developed and validated especially for complex morphology. ${ }^{9}$ In this study the GFT was selectively applied to the obstructive region in both the balloon angioplasty and the stent group. In this way, the true luminal contours could be detected.

Calibration of the image data was performed on a non-tapering part of the contrast catheter, following a similar MCA edge detection procedure as for the arterial segment; however, in this case additional information is used in the edge detection process as this part of the catheter is characterised by parallel boundaries.

From the left and right hand contours of the arterial segment a diameter function is determined. From this calculated diameter function, other variables are derived automatically; among these are percentage diameter stenosis, minimum lumen diameter, reference diameter, the area of the atherosclerotic plaque, and the extent of the obstruction.

CARDIAC AND CLINICAL END POINTS

Procedural success - The procedure was deemed to be successful if the patient left the catheter laboratory at the end of the procedure alive, with a patent vessel, less than $50 \%$ residual stenosis at the target lesion site, no clinical or electrocardiographic evidence of myocardial infarction, and did not require emergency coronary bypass surgery.

Death-This was defined as death resulting from any cause, even if not related to the target vessel angioplasty and even if not a cardiac death.

Coronary artery bypass surgery-Patients who required target vessel or non-target vessel revascularisation with coronary artery bypass surgery, either as an emergency transfer from the catheter laboratory during the initial angioplasty or electively as a further revascularisation procedure at a later time.

Vessel occlusion-Vessel occlusion was defined as occlusion occurring following the initial procedure when the patient had left the catheter laboratory. "Acute" was defined as occurring in the first 24 hours and "subacute" was defined as vessel occlusion occurring more than 24 hours but within seven days of the procedure.

Acute myocardial infarction-This was defined as a rise in the blood assay of creatine phosphokinase (CPK) to at least twice the maximum normal value or diagnostic surface ECG changes. Pathological Q waves were defined according to the Minnesota code..$^{10}$

Repeat angioplasty-This was defined as the need for a further percutaneous revascularisation procedure after the patient had left the cardiac catheter laboratory following the initial procedure.

Repeat target vessel angioplasty-This was defined if repeat angioplasty was required for the target vessel.

COMBINED CLINICAL END POINTS

Two combined clinical end points were used: the first occurrence of either death or myocardial infarction, and the first occurrence of death, myocardial infarction, or a further revascularisation procedure including repeat balloon angioplasty or coronary artery bypass grafting $(\mathrm{CABG})$.

\section{ANGIOGRAPHIC END POINTS}

Angiographic restenosis was defined as a 50\% or greater luminal narrowing compared with the reference vessel diameter.

POWER

The power calculation was based on the assumption that the angiographic restenosis rate for conventional angioplasty is $25 \%$. A sample size of 334 patients would have power of $90 \%$ to detect a reduction in the restenosis rate to $12.5 \%$ in the stent group at a significance level of $5 \%$.

\section{STATISTICAL METHODS}

The principal clinical outcome measure was the restenosis rate at six months after the procedure, and the $\chi^{2}$ test was used to compare these rates in the two treatment groups. All comparisons between outcomes in the stent and balloon angioplasty groups were made on the basis of intention to treat. Event-free survival times were analysed using the log-rank test. The continuous variables recorded from the angiographic analysis were normally distributed according to the KolmogorovSmirnov test. Angiography was performed immediately before and after the procedure and at follow up six months after the procedure. For each continuous normally distributed variable measured at angiography, a repeated measures analysis of variance was performed on the values at the three time points, with treatment group included as a factor. This provided information about whether the measure had changed over time and whether it differed between treatment groups. Angiographic results were not available for all patients at each follow up occasion, either because the patient had died or refused to have the investigation, or in some instances because it was not possible to obtain accurate estimates of the degree of stenosis from the film. The repeated measures analysis includes those patients for whom angiographic results were obtained on each of the three occasions. Post hoc $t$ tests were used to evaluate the differences 
Table 1 Patient demographics

\begin{tabular}{lccc}
\hline & POBA & Stent & Total \\
\hline Age (years) (mean (SD)) & $57.2(9.3)$ & $59.2(9.2)$ & $58.2(9.3)$ \\
Male & $111(76 \%)$ & $117(76 \%)$ & $228(76 \%)$ \\
Current smoker & $42(29 \%)$ & $27(18 \%)$ & $69(23 \%)$ \\
Ex-smoker & $75(51 \%)$ & $86(56 \%)$ & $161(54 \%)$ \\
Diabetes (insulin) & $1(1 \%)$ & $1(1 \%)$ & $2(1 \%)$ \\
Diabetes (non-insulin) & $14(10 \%)$ & $9(6 \%)$ & $23(8 \%)$ \\
Hypertension & $57(39 \%)$ & $51(33 \%)$ & $108(36 \%)$ \\
Peripheral vascular disease & $9(6 \%)$ & $4(3 \%)$ & $13(4 . \%)$ \\
Cholesterol (mmol/l) (mean (SD)) & $5.6(1.25)$ & $5.5(1.25)$ & $5.6(1.25)$ \\
CCS 0, I, and II & $77(53 \%)$ & $99(64 \%)$ & $176(59 \%)$ \\
CCS III and IV & $69(47 \%)$ & $55(36 \%)$ & $124(41 \%)$ \\
\hline
\end{tabular}

CCS, Canadian Cardiovascular Society angina grade; POBA, balloon angioplasty.

between the treatment groups at individual time points. Means were compared first for the patients who had measurements on all three occasions and then for all patients for whom measurements were available at a particular time point, to check whether these different methods of estimation produced similar results.

\section{Results}

In all, 300 patients were recruited between September 1995 and August 1997, with 146 randomised to conventional plain old balloon angioplasty (POBA group) and 154 to elective stenting with the Wiktor stent. The demographic data shown in table 1 indicate that the groups were well matched for age, sex, diabetes, hypertension, a history of peripheral vascular disease, and cholesterol level, although there was a slightly lower incidence of current smokers in the elective stent group. Table 2 shows that there was a slightly greater proportion of patients in Canadian Cardiovascular Society (CCS) class $0, \mathrm{I}$, and II in the elective stent group ( $53 \%$ v $64 \%$; NS).

The initial procedure was completed successfully in 287 of 298 patients (96\%); in two patients, both in the POBA group, the lesion did not require intervention and the procedure was aborted after initial angiographic assessment. In the 144 remaining patients in the POBA group, $44(30.1 \%)$ required deployment of a stent (37 for dissection and seven for a suboptimal result). The procedure was unsuccessful in seven patients (four were treated medically and three required surgery). In the 154 patients in the elective stent group, three $(1.9 \%)$ did not receive a stent, two because of thrombus and one because two lesions were present; the procedure was unsuccessful in four patients (two were treated medically and two required surgery). There was a considerable improvement in angina class following the procedure (table 2): at 1 year $96.3 \%$ of the 278 patients remaining in the study were in class
CCS 0, I, or II compared with $58.7 \%$ before the procedure $(\mathrm{p}<0.05) ; 58.9 \%$ of patients improved by two or more angina grades.

\section{CLINICAL EVENTS}

The clinical event rate is shown in table 3 at 30 days and in table 4 at one year. Although three deaths were recorded in the POBA group versus none in the stent group, there were no statistical differences between the two groups in any clinical events. Death and/or myocardial infarction and/or a further revascularisation procedure occurred in 10 patients $(6.8 \%)$ in the POBA group and in 12 patients $(7.8 \%)$ in the elective stent group at 30 days. Death and/or myocardial infarction at one year occurred in seven patients $(4.8 \%)$ in the POBA group and in six patients $(4.0 \%)$ in the stent group. Death and/or myocardial infarction and/or a further revascularisation procedure at one year occurred in 28 patients $(19.2 \%)$ in the POBA group and in 32 patients $(20.8 \%)$ in the elective stent group. Figure 1 shows that the event rate from six months to one year was low (POBA 5.5\%; stent 6.6\%). Acute and subacute vessel occlusion after the patient left the catheter laboratory occurred in three patients $(2.1 \%)$ in the POBA group and in five patients $(3.3 \%)$ in the stent group. Table 5 compares the event rate with that in the Benestent II study, ${ }^{4}$ showing comparable rates in the two studies.

Haemorrhage with transfusion or surgical repair of the arterial access site occurred in three patients $(2.1 \%)$ in the POBA group and in 10 patients $(6.4 \%)$ in the stent group. Of the three patients in the POBA group, two had crossed to stent deployment. These events occurred early in the trial and were associated with full anticoagulation in six of the 13 events.

\section{CLINICAL RESTENOSIS}

Table 3 shows that at 30 days nine patients $(6.1 \%)$ in the POBA group had undergone a further revascularisation procedure (repeat balloon angioplasty in five, CABG in four), and nine $(5.8 \%)$ in the elective stent group (repeat balloon angioplasty in six; CABG in four (one person underwent both procedures)). By one year, the total numbers of patients requiring a repeat revascularisation procedure were 25 in POBA group $(17.1 \%)$ and 28 in the stent group $(18.2 \%)$; the numbers requiring repeat balloon angioplasty or CABG are shown in table 3.

QUANTITATIVE ANGIOGRAPHY

Of the 300 randomised patients, 257 (86\%) underwent follow up angiography at six

Table 2 Canadian Cardiovascular Society (CCS) angina class before procedure and at six months and one year

\begin{tabular}{|c|c|c|c|c|c|c|}
\hline \multirow[b]{2}{*}{ CCS class } & \multicolumn{3}{|l|}{$P O B A$} & \multicolumn{3}{|l|}{ Stent } \\
\hline & Pre- & 6 months & 1 year & Pre- & 6 months & 1 year \\
\hline 0 & $4(2.7 \%)$ & $83(62.4 \%)$ & $103(77.4 \%)$ & $3(1.9 \%)$ & $97(66 \%)$ & $96(66.2 \%)$ \\
\hline 1 & $23(15.8 \%)$ & $30(22.6 \%)$ & $17(12.8 \%)$ & $31(20.1 \%)$ & $28(19 \%)$ & $25(17.2 \%)$ \\
\hline 2 & $50(34.2 \%)$ & $16(12.0 \%)$ & $12(9 \%)$ & $65(42.2 \%)$ & $13(8.8 \%)$ & $28(11 \%)$ \\
\hline 3 & $31(21.2 \%)$ & $4(3.0 \%)$ & $1(0.8 \%)$ & $18(11.7 \%)$ & $5(3.4 \%)$ & $7(4.8 \%)$ \\
\hline 4 & $38(26 \%)$ & $0(0 \%)$ & $0(0 \%)$ & $37(24 \%)$ & $4(2.7 \%)$ & $1(0.7 \%)$ \\
\hline Total & $146(100 \%)$ & $133(100 \%)$ & $133(100 \%)$ & $154(100 \%)$ & $147(100 \%)$ & $145(100 \%)$ \\
\hline
\end{tabular}

POBA, balloon angioplasty. 
Table 3 Severe adverse events during initial procedure

\begin{tabular}{llllc}
\hline Severe adverse event & & POBA & Stent & Total \\
\hline Death & & 2 & 0 & 2 \\
Myocardial infarction & Q wave & 2 & 0 & 2 \\
& Non-Q-wave & 1 & 6 & 7 \\
Vessel occlusion & Total & 3 & 6 & 9 \\
& Acute & 3 & 5 & 8 \\
CABG & Subacute & 1 & 0 & 1 \\
& Urgent & 2 & 4 & 6 \\
Repeat PTCA & Elective & 2 & 0 & 2 \\
& Total & 4 & 4 & 8 \\
& Target vessel & 5 & 6 & 11 \\
& Non-target vessel & 0 & 0 & 0 \\
& Total & 5 & 6 & 11
\end{tabular}

CABG, coronary artery bypass graft; POBA, balloon angioplasty; PTCA, percutaneous transluminal coronary angioplasty.

Table 4 Severe adverse events at one year

\begin{tabular}{llccc}
\hline Severe adverse event & & POBA & Stent & Total \\
\hline Death & & 3 & 0 & 3 \\
Myocardial infarction & Q wave & 2 & 1 & 3 \\
& Non-Q-wave & 3 & 5 & 8 \\
CABG & Total & 5 & 6 & 11 \\
& Urgent & 2 & 4 & 6 \\
Repeat PTCA & Elective & 4 & 3 & 7 \\
& Total & 6 & 7 & 13 \\
& Target vessel & 20 & 24 & 44 \\
\hline
\end{tabular}

months. In 20 of these, reinvestigation was not appropriate - in 11 the procedure had failed, in seven it had reached an early clinical end point, and two had not undergone the initial procedure as the lesion had regressed. A further 23 patients had refused repeat angiography, with five lost to follow up (one lost after the initial procedure and four after six months). Of the

Table 5 Widest study results against Benestent II results

\begin{tabular}{llllll}
\hline & \multicolumn{2}{l}{ PTCA } & & & \multicolumn{2}{l}{ Stent } \\
\cline { 6 - 6 } \cline { 5 - 6 } \cline { 5 - 6 } & Widest & Benestent II & & Widest & Benestent II \\
\hline Crossover & $30.1 \%$ & $13.4 \%$ & & $1.9 \%$ & $3.4 \%$ \\
FU angiogram & $83 \%$ & $51 \%$ & & $90 \%$ & $50.1 \%$ \\
Restenosis rate & $17.3 \%$ & $31 \%$ & & $21.6 \%$ & $16 \%$ \\
Early events & $6.8 \%$ & $5.1 \%$ & & $7.8 \%$ & $3.9 \%$ \\
One year events & $19.2 \%$ & $23.4 \% \star$ & & $20.8 \%$ & $20.7 \% \star$ \\
\hline
\end{tabular}

${ }^{\star}$ In angiogram follow up patients.

FU, follow up.

Table 6 Comparison of quantitative coronary angiography data between the two groups

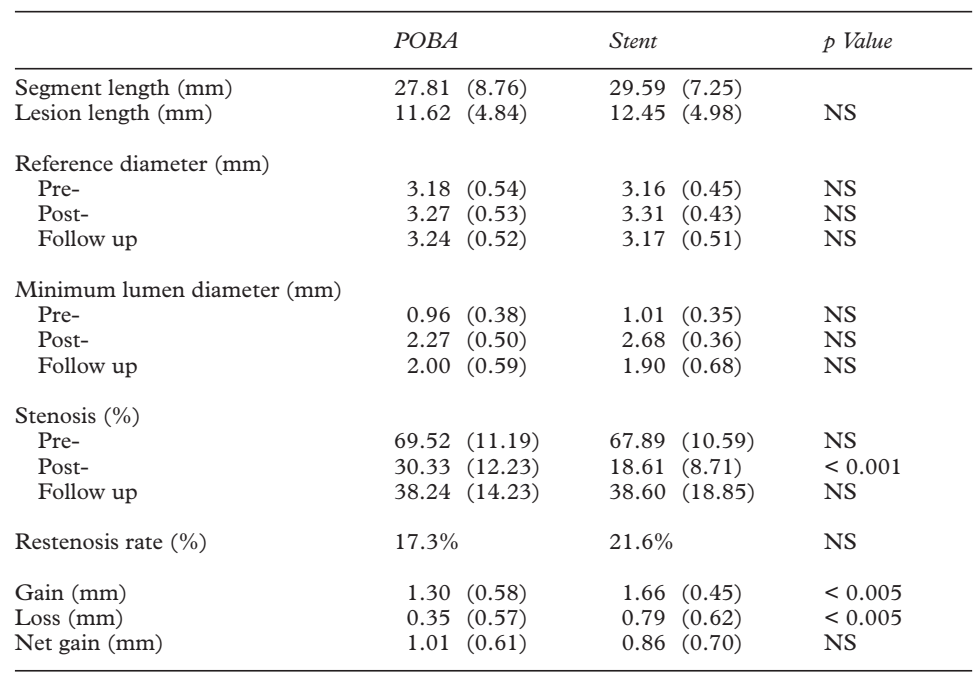

Values are mean (SD) unless stated otherwise.

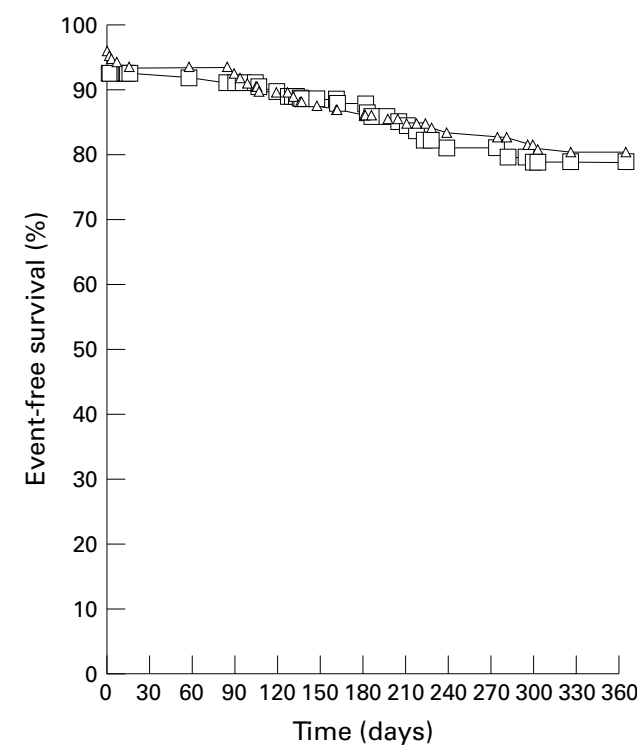

Figure 1 Kaplan-Meier curve curve showing event-free survival (\%) from death, myocardial infarction, or a repeat revascularisation procedure. $\square$, stent; $\triangle, P O B A$.

257 follow up angiograms done, 213 were analysed ( $71 \%$ in all; POBA $69 \%$, stent $73 \%$ ), as 44 patients were excluded for technical reasons. Sixty seven patients who should therefore have undergone quantitative coronary angiography did not do so. Of this group, seven underwent a further revascularisation procedure (balloon angioplasty in four; stent in three), and one patient in the POBA group suffered a myocardial infarct at the initial procedure. The event rate in this group was therefore $12.9 \%$ at one year, compared with $18.8 \%$ in the group that had undergone angiographic follow up and analysis. In the remaining 59 patients, only one was classified as CCS III, with no patients in CCS IV. No events were recorded in the group refusing angiographic follow up.

Table 6 summarises the data from the quantitative coronary angiography. The groups were well matched before intervention for minimum lumen diameters (POBA $0.96 \mathrm{~mm}$; stent 1.01 $\mathrm{mm})$. Following intervention the stent group had a greater improvement in minimum lumen diameter than the POBA group (POBA 2.27 $\mathrm{mm} v$ stent $2.68 \mathrm{~mm}$; $\mathrm{p}<0.007)$. However, late loss was greater in the stent group, and at six months there was no difference between the groups (POBA $2.00 v$ stent 1.90; p > 0.05), while the angiographic restenosis rate was not significantly different (POBA $17.3 \% v$ stent $21.6 \% ; \mathrm{p}>0.05)$. Table 7 shows that the patients who crossed over from POBA to stent group behaved like the ones in the elective stent group, with an initially greater gain and a subsequently greater loss. Table 5 shows that, compared with the Benestent II study, it was not the failure of the stent group to perform well but the superior performance of the POBA group - with its higher rate of crossover than in Benestent- that resulted in there being no difference between the two groups. 
Table 7 Comparison of quantitative coronary angiography data between patients in the POBA group who crossed over and those who did not, compared with the stent group

\begin{tabular}{|c|c|c|c|c|c|c|c|}
\hline \multirow{3}{*}{$\begin{array}{l}\text { Segment length }(\mathrm{mm}) \\
\text { Lesion length }(\mathrm{mm})\end{array}$} & \multicolumn{2}{|c|}{$\begin{array}{l}\text { POBA, } \\
\text { crossover }\end{array}$} & \multicolumn{2}{|c|}{$\begin{array}{l}\text { POBA, no } \\
\text { crossover }\end{array}$} & \multicolumn{2}{|l|}{ Stent } & \multirow[t]{2}{*}{$p$ Value } \\
\hline & 30.78 & $(9.34)$ & 26.55 & $(8.23)$ & 29.59 & $(7.25)$ & \\
\hline & 12.02 & $(5.27)$ & 11.46 & $(4.67)$ & 12.45 & $(4.98)$ & NS \\
\hline \multicolumn{8}{|l|}{ Reference diameter (mm) } \\
\hline Pre- & 3.20 & $(0.42)$ & 3.17 & $(0.58)$ & 3.16 & $(0.45)$ & NS \\
\hline Post- & 3.37 & $(0.43)$ & 3.22 & $(0.57)$ & 3.31 & $(0.43)$ & NS \\
\hline Follow up & 3.24 & $(0.41)$ & 3.23 & $(0.56)$ & 3.17 & $(0.51)$ & NS \\
\hline \multicolumn{8}{|c|}{ Minimum lumen diameter (mm) } \\
\hline Pre- & 0.93 & $(0.30)$ & 0.97 & $(0.41)$ & 1.01 & $(0.35)$ & NS \\
\hline Post- & 2.69 & $(0.41)$ & 2.08 & $(0.42)$ & 2.68 & $(0.36)$ & $<0.005$ \\
\hline Follow up & 2.15 & $(0.42)$ & 1.93 & $(0.65)$ & 1.90 & $(0.68)$ & NS \\
\hline \multicolumn{8}{|l|}{ Stenosis (\%) } \\
\hline Pre- & 70.56 & $(9.03)$ & 69.08 & $(12.01)$ & 67.89 & $(10.59)$ & NS \\
\hline Post- & 19.98 & $(8.01)$ & 34.85 & $(10.97)$ & 18.61 & $(8.71)$ & $<0.005$ \\
\hline Follow up & 33.58 & $(10.10)$ & 40.06 & $(15.22)$ & 38.60 & $(18.85)$ & NS \\
\hline Restenosis rate (\%) & $13.5 \%$ & & $22.9 \%$ & & $21.6 \%$ & & NS \\
\hline Gain (mm) & 1.75 & $(0.47)$ & 1.11 & $(0.51)$ & 1.66 & $(0.45)$ & $<0.005$ \\
\hline Loss (mm) & 0.61 & $(0.50)$ & 0.23 & $(0.57)$ & 0.79 & $(0.62)$ & $<0.005$ \\
\hline Net gain $(\mathrm{mm})$ & 1.22 & $(0.49)$ & 0.92 & $(0.64)$ & 0.86 & $(0.70)$ & 0.004 \\
\hline
\end{tabular}

Values are mean (SD) unless stated otherwise.

\section{Discussion}

Coronary angioplasty is a highly effective method of relieving angina caused by coronary artery stenosis. The results in terms of symptomatic improvement in this trial are consistent with the findings elsewhere and are substantially better than would be expected for medical treatment alone, although slightly less good than after coronary bypass surgery. The absence of any significant difference between the angioplasty and stent groups confirms that the benefit relates to the quality of the result in terms of increase in lumen diameter and relief of obstruction rather than to the specific technology used.

Before the introduction of coronary stents, an inverse relation between the postprocedural minimum lumen diameter and the restenosis rate had been shown, ${ }^{11}$ although it was also known that the principle of "greater acute gain, greater late loss" also applied. Enthusiasm for aggressive dilatation, or even oversizing, has been tempered by the increased risk of acute vessel occlusion and its associated complications. The Benestent and STRESS (stent restenosis study) trials showed that the deployment of coronary stents leads to a greater initial luminal gain. However, late loss appeared to be greater than with balloon angioplasty so that at six months there was little difference between the two approaches. ${ }^{45}$ In our study there was no advantage of an elective stent strategy over balloon angioplasty, although - with a crossover rate of $30 \%$ - a more accurate title for the POBA group would be "provisional stent strategy." Comparing the event rates and the quantitative angiography data with those of the Benestent II study, ${ }^{12}$ it can be seen that the good results with our POBA group were associated with a low event and restenosis rate and a higher initial lumen gain. This presumably reflects the more aggressive angioplasty strategy in current use, now that it is known that vessel dissection and acute vessel closure can be effectively treated by deployment of a stent. Advances in balloon technology and a better understanding of the mechanical consequences of balloon inflation at high pressure may also contribute to the improvement in angioplasty results.

The Wiktor stent is a tantalum wire stent with a substantially more open mesh than laser cut slotted tube stents. There is some evidence that intimal protrusion may be more common with this type of open stent, ${ }^{13}$ and compared with the Benestent II data the early event rate is higher, although by one year there is little difference between the two studies. ${ }^{12}$ The discrepancy may also be explained by our sparing use of ticlopidine and abciximab, although comparable early event rates have been seen in other recent studies. ${ }^{14}$ The advantages of the more open structure, namely less risk of "jailing" of side branches and greater flexibility and conformity in tortuous segments of artery, justify the use of this type of stent, which, although not commonly employed in the United Kingdom, is extensively used elsewhere.

At the time of initial recruitment into this study the conventional treatment following stent deployment was the use of an intensive regimen of full anticoagulation combined with aspirin. Soon after the start of the trial, effective antiplatelet drugs became available ${ }^{1516}$ but were not licenced for use in the United Kingdom. The poststent drug regimen was therefore deliberately not specified, in order to allow centres to use their normal drug combination. However, this did produce some distortion in the results, particularly with regard to early complication rates. Haemorrhage, transfusion, or surgical repair of the arterial puncture site was almost entirely confined to patients receiving full anticoagulation, the majority being in the stent group (10 of the 13). Two of the three patients in the POBA group who had experienced this complication had undergone stent deployment. There was a clear trend throughout the trial away from this type of treatment. Towards the end of the trial virtually all patients were receiving the combination of aspirin and ticlopidine rather than full anticoagulation, and very few haemorrhagic episodes were recorded at this stage.

The use of a full anticoagulant regimen has subsequently been shown to have adverse effects in terms of acute vessel occlusion compared with an intensive antiplatelet regimen. ${ }^{15} 16$ The comparably high acute and subacute occlusion rate in our study can be accounted for by this phenomenon. Abciximab was not available for most of the period of recruitment and only one patient, towards the end of the trial, received this treatment.

Although it was originally intended to recruit 400 patients to this study, the trial was terminated prematurely after 300 patients had been enrolled. This was close to the number indicated by the original power calculation, and the decision to terminate early was taken because of a declining recruitment rate, as many other technologies came (and, in some cases, went), causing some centres to have difficulty in maintaining recruitment. The results, 
however, clearly indicate that the early termination did not affect the overall evaluation of the outcomes.

The similarity of the results in the angioplasty and stent groups was related to the high rate of crossover to stent deployment in the angioplasty group. The patients who crossed over achieved luminal gains comparable with those in the elective stenting group and greater than those who did not cross over; inevitably this raises the question of what is the ideal stenting rate. At one end of the scale it can be argued that a policy of elective stenting whenever technically possible will guarantee the optimum lumen gain and the greatest chance of long term benefit. On the other hand the results suggest that a policy of provisional stenting can achieve just as good an overall long term results with the cost savings that may result from lower stent usage. Further studies to elucidate this are clearly essential and these should take into account the varying stent rates in different centres at present.

This study involved a selected group of patients and, because of the multicentre and multinational nature of the trial, the precise degree of selection was impossible to evaluate with accuracy. This makes assessment of the clinical context of these findings more difficult, although the inclusion criteria were such as to potentially include a substantial proportion of patients undergoing elective angioplasty. We urgently need a prospective analysis of the relative overall costs of provisional versus elective stenting and the respective gains in terms of symptomatic improvement, employment status, and quality of life when either strategy is used.

The Widest trial investigators' group included: Drs Bucknall and Roper (Guy's and St Thomas' Hospital, London); Drs Dymond and Hogan (St Bartholomew's Hospital, London); Dr Dymond and Hogan (St Bartholomew's Hospital, London); Dr Campbell (Northern General Hospital, Sheffield); Dr Quigley (St Vincent's Hospital, Dublin, Republic of Ireland); Dr Brecke Hospital, London). Members of the safety monitoring committee were: Dr N Brooks (Chairman, Wythenshawe Hospital,
Manchester), Dr M Vrolix (St Jan Hospital, Scheapsa, NetherManchester), Dr M Vrolix (St Jan Hospital, Scheapsa, Nether-
lands), and Dr S Davies (Royal Brompton Hospital, London).

1 Gruentzig AR, Senning A, Siegenthaler WE. Non-operative dilatation of coronary artery stenosis-percutaneous transdilatation of coronary artery stenosis-percutaneous trans-
luminal coronary angioplasty. $N$ Engl $\mathscr{f}$ Med 1979;301:61.

luminal coronary angioplasty. N Engl f Med 1979;301:61.
2 Hillegas W, Ohman O, Califf R. Restenosis: the clinical Hillegas W, Ohman O, Califf R. Restenosis: the clinical
issues. In: Jopol EJ. ed. Textbook of interventional cardiology, issues. In: Jopol EJ. ed. Textbook of interventional card
vol 2. Philadelphia: W B Saunders, 1994:415-35.

3 Sigwart U, Kaufmann U, Golf S, et al. [[Incidence and treatment of coronary restenosis in spite of the implantation of an endoprosthesis.]] Schweiz Med Wochenschr 1988; 118: $1715-18$

4 Serruys PW, de Jaegere P, Kiemeneij F, et al. A comparison of balloon-expandable-stent implantation with balloon angioplasty in patients with coronary artery disease. Benestent Study Group. N Engl f Med 1994;331:489-95.

5 Fischman DL, Leon MB, Baim DS, et al. A randomized comparison of coronary-stent placement and balloon angioplasty in the treatment of coronary artery disease. Stent restenosis stud

6 Narins CR, Holmes DR, Topol EJ. A call for provisional Narins CR, Holmes DR, Topol EJ. A call for provisional
stenting: the balloon is back! Circulation 1998;97:1298305.

7 Reiber JHC, von Land CD, Koning G, et al. Comparison of accuracy and precision of quantitative coronary arterial analysis between cinefilm and digital systems. In: Reiber J H C, Serruys P W, eds. Progress in quantitative coronary arteriography. Dordrecht: Kluwer, 1994:67-85.

8 Reiber JHC, Schiemanck LR, Zwet PMJvd, et al. State of the art in quantitative coronary arteriography as of 1996. In: Reiber J H C, van der Wall E E, eds. Cardiovascular imaging. Dordrecht: Kluwer, 1996:39-56.

9 van der Zwet PM, Reiber JH. A new approach for the quantification of complex lesion morphology: the gradient field transform; basic principles and validation results. $\mathcal{f} \mathrm{Am} \mathrm{Coll}$ Cardiol 1994;24:216-24.

10 Blackburn H, Keys A, Simonson E, et al. The electrocardiogram in population studies: a classification system. Circulation 1960;21:1160-75.

11 Kuntz RE, Gibson CM, Nobuyoshi $M$, et al. Generalized model of restenosis after conventional balloon angioplasty, model of restenosis after conventional balloon angioplasty, 1993;21:15-25.

12 Serruys PW, van Hout B, Bonnier H, et al. Randomised comparison of implantation of heparin-coated stents with balloon angioplasty in selected patients with coronar artery disease (Benestent II). Lancet 1998;352:673-81.

13 Buchwald A, Unterberg C, Werner G, et al. Initial clinical results with the Wiktor stent: a new balloon-expandable coronary stent. Clin Cardiol 1991;14:374-9.

14 The EPISTENT Investigators. Randomised placebocontrolled and balloon-angioplasty-controlled trial to assess safety of coronary stenting with use of platelet glycoprotein-IIb/IIIa blockade. Lancet 1998;352:87-92.

15 Colombo A, Hall P, Nakamura S, et al. Intracoronary stenting without anticoagulation accomplished with intravascular ultrasound guidance Circulation 1995;91:1676-88.

16 Hall P, Nakamura S, Maiello L, et al. A randomized comparison of combined ticlopidine and aspirin therapy versus aspirin therapy alone after successful intravascular ultrasound-guided stent implantation. Circulation 1996;93: 215-22. 